\title{
Relevance of serum angiogenic cytokines in adult patients with dermatomyositis
}

\author{
Thiago Costa Pamplona da Silva, Marilda Guimarães Silva and Samuel Katsuyuki Shinjo*
}

\begin{abstract}
Background: Until now, there are few studies evaluating serum levels of angiogenic cytokines in dermatomyositis (DM). Therefore, the aims of the present study were: (a) to analyze systematically and simultaneously serum levels of angiogenin (ANG), angiopoietin (ANGPT)-1, vascular endothelial growth factor (VEGF), fibroblast growth factor (FGF)1 and -2, platelet derived growth factor (PDGF)-AA and -BB in DM; (b) to correlate the serum level of these cytokines with the DM clinical and laboratory features.

Methods: This is a cross sectional study, in which 48 patients with DM aged 18 to 45 years were gender-, age- and ethnicity-matched with 48 healthy individuals (control group). The serum levels of cytokines analyses were performed by multiplex immunoassay. The parameters of DM activity were based on the scores established by the International Myositis Assessment \& Clinical Studies Group.
\end{abstract}

Results: The mean ages, gender frequencies and ethnicities were comparable between the patients with DM and the control group. A significantly higher serum FGF-1 and FGF-2 levels $(P<0.001$ and $P<0.001$, respectively), lower VEGF and PDGF-AA levels $(P=0.009$ and $P=0.022)$, and comparable ANG, ANGPT-1 and PDGF-BB levels were observed in DM patients compared to controls. There was a tendency for cytokines (with the exceptions of VEGF and PDGF-BB) to correlate positively with the DM activity parameters, whereas FGF-2 correlated inversely. Moreover, FGF-1 strongly correlated with DM cutaneous manifestations.

Conclusions: The present data provide the relevance of different serum angiogenic cytokines in patients with DM. Additional studies will be needed to validate the data obtained in this work.

Keywords: Angiogenesis, Cytokines, Dermatomyositis, Idiopathic inflammatory myopathies, Myositis

\section{Background}

Dermatomyositis (DM) is an autoimmune inflammatory myopathy characterized by a subacute onset and progressive skeletal muscle weakness. The disease is associated with typical cutaneous manifestations, including heliotrope and/or Gottron's papules [1-7]. The cornerstone of DM physiopathogenesis involves vascular disturbances and a primarily humoral immune response [4-6], and can involve multiple cytokines related to mechanisms ranging from inflammation to angiogenesis.

Several serum angiogenic cytokines have been described in the literature, including: angiogenin (ANG), angiopoietin-1 (ANGPT-1), vascular endothelial growth factor (VEGF), fibroblast growth factor (FGF) types 1

\footnotetext{
* Correspondence: samuel.shinjo@gmail.com

Division of Rheumatology, Faculdade de Medicina FMUSP, Universidade de

Sao Paulo, Av. Dr. Arnaldo, 455, $3^{\circ}$ andar, sala 3150 - Cerqueira César, Sao

Paulo CEP: 01246-903, Brazil
}

(c) The Author(s). 2018 Open Access This article is distributed under the terms of the Creative Commons Attribution 4.0 International License (http://creativecommons.org/licenses/by/4.0/), which permits unrestricted use, distribution, and reproduction in any medium, provided you give appropriate credit to the original author(s) and the source, provide a link to the Creative Commons license, and indicate if changes were made. The Creative Commons Public Domain Dedication waiver (http://creativecommons.org/publicdomain/zero/1.0/) applies to the data made available in this article, unless otherwise stated. PDGF-BB). However, these serum angiogenic cytokines have been scarcely assessed in DM [8-14]. For instance, Kuwahara [8] observed high ANG mRNA expression in the skin tissue of patients with active DM but no significant increase in the serum ANG level. Nevertheless, the authors described a positive correlation between the serum ANG and aldolase levels.

High serum VEGF levels have been noted in DM and polymyositis (PM) [9-11]. However, as a limitation, these data are based on a series of cases, involving individuals mostly $\geq 40$ years of age with no detailed treatment information.

Kadono et al. [13] observed an elevated serum FGF-2 level in DM patients that correlated with the serum creatine phosphokinase (CPK) level and pulmonary fibrosis. 
However, this study assessed only 7 untreated female patients $\geq 50$ years of age.

The serum ANGPT-1 levels were normal in patients with $\mathrm{DM}$ in a unique study in the literature [12]; however, although additional information concerning the association between values and clinical or laboratory parameters was provided, no date concerning the serum FGF-1 and PDGFs, there are no data evaluating serum levels were available for these patients.

Therefore, the aim of the present research was to assess simultaneously and systematically the serum ANG, ANGPT-1, VEGF, FGF (types 1 and 2), PDGF-AA and PDGF-BB levels in DM patients. Additionally, we sought to correlate the serum levels of these cytokines with demographic, clinical, laboratory, and therapeutic factors and comorbidities of patients with DM.

\section{Methods}

A cross-sectional study was performed at a single centre. The study included 60 consecutive patients with DM (age $\geq 18$ and $\leq 45$ years) enrolled from 2012 to 2014 who fulfilled all of the Bohan and Peter criteria items [1,2] and were regularly followed at our outpatient myopathy unit. Patients with cancer-associated myositis, clinically amyopathic DM and overlapped myositis were not included in the study.

The study was approved by the local ethics committee (HCFMUSP - CAPPesq number 1.545.393) and all patients signed the informed consent form.

To avoid possible factors that could interfere with serum cytokine analysis, patients with $>10$ years of DM disease $(n=8)$ and tobacco habits $(n=4)$ were excluded. Moreover, no cases of acute and/or chronic infections, liver and renal diseases, menopause, diabetes mellitus, non-controlled chronic systemic arterial hypertension, myocardial infarction, ischemic stroke, alcohol consumption and claudication vascular symptoms were included. Therefore, 48 patients with DM were assessed, and 48 age, gender and ethnicity-matched healthy volunteers were recruited as a control group during the same period.

All participants underwent a clinical evaluation that included a standardized interview, and their charts were extensively reviewed.

Demographic data were collected, including the current age, gender, ethnicity, waist circumference, weight and body mass index [BMI, weight $/$ height $\left.{ }^{2}\left(\mathrm{~kg} / \mathrm{m}^{2}\right)\right]$. The clinical and laboratory data included the age at disease onset, disease duration, muscle enzyme serum levels [CPK: reference value 26-308 U/L, aldolase: $<7.5 \mathrm{U} / \mathrm{L}$, alanine aminotransferase (ALT): < $41 \mathrm{U} / \mathrm{L}$, aspartate aminotransferase (AST): <37 U/L, lactate dehydrogenase (LDH): 240-480 U/L)], and clinical manifestations [articular (arthralgia or arthritis), pulmonary (moderated or severe subjective dyspnea associated simultaneously with confirmed "ground-glass" on high-resolution chest computed tomography) activity, and cutaneous (Gottron's papules, heliotrope rash, ulcers, vasculitis, "shawl" sign, "V-neck" sign, facial rash, Raynaud's phenomenon, and calcinosis].

The disease status was evaluated using the following questionnaires and scores: global assessment of the disease (by the physician and the patient) through the visual analogue scale (VAS) [15-17], Manual Muscle Testing (MMT-8) [18], Health Assessment Quality (HAQ) [19].

Therapy data included the use of immunosuppressives and, glucocorticoids (current and cumulative doses).

Cytokine assessment. A blood sample (10 mL of blood) obtained from each participant after a 12-h overnight fast was immediately $(<30 \mathrm{~min})$ centrifuged at $3000 \mathrm{rpm}$ for 10 min at $4{ }^{\circ} \mathrm{C}$. The serum was stored at $-80^{\circ} \mathrm{C}$ prior analysis of the cytokines ANG, ANGPT-1, VEGF, FGF-1, FGF-2, PDGF-AA and PDGF-BB. The analysis was performed using the Luminex 200- xMAP Technology (Millipore, USA), as described elsewhere [20].

Statistical analysis. The Kolmogorov-Smirnov test was used to evaluate the distribution of each parameter. The demographic and clinical features were expressed as the mean \pm standard deviation (SD) for continuous variables or as frequencies and percentages (\%) for categorical variables. The median (25th - 75th interquartile range) was calculated for continuous variables that were non-normally distributed. Comparisons between the patient and control parameters were made using Student's $t$-test or the Mann-Whitney test for continuous variables, whereas the Chi-square test or Fisher's exact test was used to evaluate categorical variables. The correlations among the parameters were analysed by Spearman's correlation. All of the analyses were performed using the SPSS 15.0 statistical software (Chicago, USA). A $P<0.05$ was considered to indicate statistical significance.

\section{Results}

Forty-eight patients with DM and 48 controls were evaluated. As expected, the mean age, gender frequency and ethnicity were similar between the groups (Table 1). The mean age at disease onset was 30.9 years, with 5.0 months of symptoms prior to diagnosis and median disease duration of 1.0 year.

The articular, pulmonary and cutaneous involvements were present in 29.2, 35.4 and $100 \%$ of the cases, respectively.

The disease status parameters are shown in Table 1. As expected, all muscle enzymes were significantly higher in the patients with DM than in the controls. 
Table 1 General features of patients with dermatomyositis and health individuals

\begin{tabular}{|c|c|c|c|}
\hline Parameters & $\mathrm{DM}(n=48)$ & Control $(n=48)$ & $P$ value \\
\hline Age (years) & $33.3 \pm 7.6$ & $35.8 \pm 8.2$ & 1.000 \\
\hline White ethnicity & $36(75.0)$ & $32(66.7)$ & 0.501 \\
\hline Female gender & $36(75.0)$ & $36(75.0)$ & 1.000 \\
\hline Age at disease onset (years) & $30.9 \pm 7.4$ & - & - \\
\hline Duration: diagnosis - symptoms (months) & $5.0(2.3-8.5)$ & - & - \\
\hline Disease duration (years) & $1(0-4)$ & - & - \\
\hline \multicolumn{4}{|l|}{ Clinical cumulative manifestations } \\
\hline Articular involvement & $14(29.2)$ & - & - \\
\hline Pulmonary involvement & $17(35.4)$ & - & - \\
\hline Cutaneous involvement & $48(100.0)$ & - & - \\
\hline Gottron's papules & $47(97.9)$ & - & - \\
\hline Heliotrope rash & $40(83.3)$ & - & - \\
\hline Facial rash & $30(62.5)$ & - & - \\
\hline Raynaud' phenomenon & $24(50.0)$ & - & - \\
\hline "V-neck" sign & $15(31.3)$ & - & - \\
\hline Ulcers & $10(20.8)$ & - & - \\
\hline Vasculitis & $10(20.8)$ & - & - \\
\hline "Shawl" sign & $8(16.7)$ & - & - \\
\hline Calcinosis & 0 & - & - \\
\hline MMT-8 (0-80) & $78(71-80)$ & & \\
\hline HAQ (0.00-3.00) & $0.36(0.00-2.00)$ & - & - \\
\hline Patient VAS (0-10 mm) & $5(1-6)$ & - & - \\
\hline Physician VAS (0-10 mm) & $4(1-5)$ & - & - \\
\hline Creatine phosphokinase $(U / L)$ & $200(93-960)$ & $100(81-161)$ & 0.002 \\
\hline Aldolase (U/L) & $5.9(4.1-12.9)$ & $3.6(3.1-4.3)$ & $<0.001$ \\
\hline Lactic dehydrogenase (U/L) & $412(347-597)$ & $323(296-381)$ & $<0.001$ \\
\hline Alanine aminotransferase (U/L) & $25(16-60)$ & $17(13-21)$ & $<0.001$ \\
\hline Aspartate aminotransferase $(U / L)$ & $25(19-52)$ & $19(16-22)$ & $<0.001$ \\
\hline \multicolumn{4}{|l|}{ Prednisone } \\
\hline Current use & $34(70.8)$ & - & - \\
\hline Current dose (mg/day) & $20.0(3.1-50.0)$ & - & - \\
\hline Cumulative dose $\mathrm{a}^{\mathrm{a}}(\mathrm{mg})$ & $645(90-2103)$ & - & - \\
\hline Cumulative dose $\mathrm{e}^{\mathrm{b}}(\mathrm{g})$ & $15.5(5.8-27.8)$ & - & - \\
\hline Immunosupressive/imunmodulatory ${ }^{c}$ & & & - \\
\hline None & $20(41.7)$ & - & - \\
\hline One & $11(22.9)$ & - & - \\
\hline Two & $17(35.4)$ & - & - \\
\hline
\end{tabular}

Results expressed as percentage (\%), mean \pm standard deviation, median (25th - 75th interquartile range)

DM dermatomyositis, HAQ Health Assessment Questionnaire, MMT Muscle Manual Testing, VAS visual analogue score

${ }^{\mathrm{a}}$ Last 3 months; ${ }^{\mathrm{b}}$ since the begin of treatment; ${ }^{\mathrm{c}}$ imunossupressive / imunomodulatory: azathioprine (2-3 mg/kg/day), methotrexate (15-25 mg/week),

cyclosporine ( $1.5-2.5 \mathrm{mg} / \mathrm{kg} /$ day), mycophenolate mofetil $(2-3 \mathrm{~g} /$ day), rituximab $(1 \mathrm{~g}$, intravenous, at baseline and after one month - first cycle - and this schema was repeated after six months), cyclophosphamide (0.5-1.0 g/ $\mathrm{m}^{2}$ body surface), leflunomide ( $20 \mathrm{mg} / \mathrm{day}$ ) and/or intravenous human immunoglobulin ( $2 \mathrm{~g} / \mathrm{kg}$, daily, two consecutive days)

Regarding drug treatment, $70.8 \%$ of the patients were using prednisone with a mean dose of $20.0 \mathrm{mg} /$ day. The median cumulative dose of prednisone was $645 \mathrm{mg}$ (over the last three months of blood collection) and $15.5 \mathrm{~g}$ (since the onset of disease symptoms). Approximately half of the patients were using at least 
one immunosuppressive or immunomodulatory drug, including azathioprine $(2-3 \mathrm{mg} / \mathrm{kg} /$ day $)$, methotrexate (15-25 mg/week), cyclosporine (1.5-2.5 mg/kg/day), mycophenolate mofetil (2-3 g/day), rituximab [1 g, intravenous, at baseline and after one month (first cycle); this scheme was repeated after six months], cyclophosphamide $\left(0.5-1.0 \mathrm{~g} / \mathrm{m}^{2}\right.$ body surface), leflunomide $(20 \mathrm{mg} /$ day $)$ and/or human intravenous immunoglobulin (2 g/kg, daily, two consecutive days) (Table 1).

The serum levels of the angiogenic cytokines (ANG, ANGPT-1 and PDGF-BB) were comparable between the groups (Table 2). The FGF-1 and FGF-2 levels were elevated, whereas the VEGF and PDGF-AA levels were decreased in the patients with DM compared to the control group.

Table 3 shows only the significant correlations between the angiogenic cytokines analysed in the present study and the demographic, clinical, laboratory and therapeutic parameters shown previously in Table 1 . Moreover, the correlations between the cytokines themselves were also analysed. All data refer to patients with DM.

The FGF-1 serum levels were moderately correlated with the cutaneous clinical manifestations (facial rash, "V-neck" sign and "shawl" sign) as well as some disease activity parameters (patient and physician VAS, and muscle enzymes) and were inversely correlated with the disease duration and cumulative prednisone dose. Additionally, the serum FGF-1 levels positively correlated with the serum ANG, ANGPT-1 and PDGF-AA levels and negatively correlated with the FGF-2 levels.

FGF-2 tended to be inversely correlated with the disease activity parameters (patient and physician VAS, MMT-8, and muscle enzymes) and serum FGF-1, ANG, ANGPT-1, PDGF-AA and PDGF-BB levels. A positive correlation was found between the serum FGF-2 levels and the cumulative prednisone dose.

Table 2 Serum levels of angiogenic cytokines in patients with dermatomyositis and healthy individuals

\begin{tabular}{llll}
\hline Parameters & DM $(\mathrm{n}=48)$ & Control $(\mathrm{n}=48)$ & $P$ value \\
\hline ANG $(\mathrm{ng} / \mathrm{mL})$ & $4383(3588-7251)$ & $3931(2735-5397)$ & 0.138 \\
ANGPT-1 $(\mathrm{ng} / \mathrm{mL})$ & $8576(4964-13,060)$ & $8080(5785-9609)$ & 0.224 \\
VEGF $(\mathrm{ng} / \mathrm{mL})$ & $28.6(5.8-49.3)$ & $38.6(21.3-72.6)$ & 0.009 \\
FGF-1 $(\mathrm{ng} / \mathrm{mL})$ & $3.1(1.8-14.9)$ & $0.7(0.4-1.8)$ & $<0.001$ \\
FGF-2 $(\mathrm{ng} / \mathrm{mL})$ & $1.6(0.6-3.1)$ & $0.3(0.0-0.9)$ & $<0.001$ \\
PDGF-AA $(\mathrm{ng} / \mathrm{mL})$ & $545(304-797)$ & $654(411-1083)$ & 0.022 \\
PDGF-BB $(\mathrm{ng} / \mathrm{mL})$ & $2039(1539-2506)$ & $2107(1752-2465)$ & 0.358
\end{tabular}

Results expressed as median (25th - 75th interquartile range)

ANG angiogenin, ANGPT-1 angiopoietin-1, DM dermatomyositis, FGF fibroblast growth factor, PDGF platelet-derived growth factor, VEGF vascular endothelial growth factor
Low serum PDGF-AA and VEGF levels were observed in patients with DM. No correlation was found between the VEGF level and the disease activity parameters or treatment data. However, the serum PDFG-AA, and FGF-1 levels were positively correlated with the DM disease parameters (physician VAS, MMT-8, HAQ, and muscle enzymes) and inversely correlated with the disease duration and cumulative prednisone dose. A positive correlation between the PDGF level and the serum ANG, ANGPT-1 and PDGF-AA levels and a negative correlation with the FGF-2 level were also observed.

ANG, ANGPT-1 and PDGF-BB were not elevated in DM. However, the correlations among the serum levels of these cytokines and the other parameters generally followed the same profiles observed for FGF-1 and PDGF-AA.

\section{Discussion}

This study is the first to systematically and simultaneously analyse the serum levels of several angiogenic cytokines in patients with DM.

The great advantage of the present study was its use of rigorous selection criteria for patients. Additionally, we excluded confounding factors that could interfere with the evaluation and interpretation of the angiogenic cytokines.

FGF-1 was positively correlated with the cutaneous clinical manifestations and some disease activity parameters, which was in contrast with FGF-2. We also observed lower FGF-1 levels in the patients with longer disease duration and a cumulative utilization of prednisone.

Both FGF-1 and -2 act in several cellular processes and specifically, in angiogenesis, they induce cell proliferation and the physical organization of endothelial cells into tubular structures [21-24]. Due to their specific biological functions and roles, FGFs have the potential for application to induce the regeneration of a wide spectrum of tissues [24].

By combining these known actions of FGF-1 with our findings, we propose that this cytokine may be involved in the physiopathogenesis of cutaneous manifestations in active DM patients, because patients with more cutaneous lesions, a shorter duration of disease or long-term treatment have higher serum FGF-1 levels. Further studies of cutaneous biopsies to evaluate local levels of FGF-1 or its genic expression could corroborate this hypothesis.

FGF-2 has a more powerful healing action than FGF-1. Indeed, blockage of FGF-2 activity is almost completely impairs wound angiogenesis [25]. Moreover, some in vitro and in vivo studies have demonstrated that FGF-2 promotes stem cell recruitment during the muscle regeneration process [26]. 
Table 3 Spearman's correlation between angiogenic cytokines and diferent disease parameters

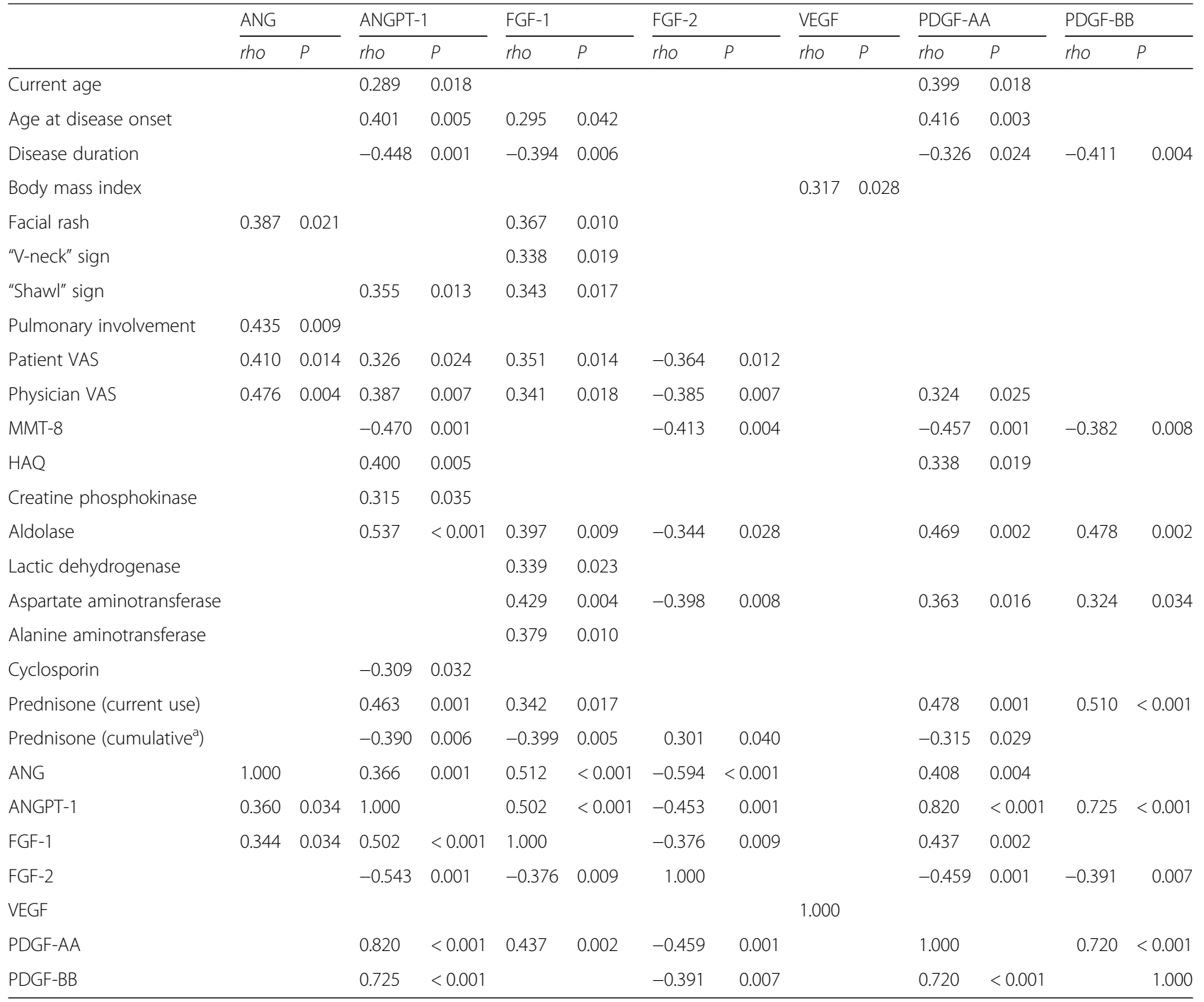

ANG angiogenin, ANGPT-1 angiopoietin-1, FGF fibroblast growth factor, HAQ Health Assessment Questionnaire, MMT Muscle Manual Testing, $P P$ value, $P D G F$ platelet-derived growth factor-AA and BB; Rho: Spearmen's correlation, VAS visual analogue scores, VEGF vascular endothelial growth factor

${ }^{a}$ Since the disease onset symptoms

A negative correlation between the serum FGF-2 level and the disease activity parameters and a positive correlation with the cumulative prednisone dose were noted. One possible explanation for this finding is that FGF-1 and FGF-2 are continuously released into the bloodstream by muscle and endothelial tissues due to myofibrillar necrosis. Alternatively, the muscle regeneration process and fibrosis that occur during the evolution of disease or its treatment may maintain the elevated FGF-2 level due to its potent cure and regeneration actions. This phenomenon would make FGF-2 a marker of the muscular healing process in DM patients.

We found an inverse pattern of correlation between FGF-1 and -2 (positive and negative, respectively) and the other angiogenic cytokines evaluated. We conclude that there are mechanisms that up and down-regulate
FGF-1 and FGF-2 during the different stages of disease progression with a more established counter-regulation in chronic or adequately treated cases.

PDGF-AA and PDGF-BB promote the maturation of blood vessels through the recruitment and adhesion of mural cells by specific interactions with their receptors PDGF-R $\alpha \alpha$ (positive mitotic signals) and PDGF-R $\beta \beta$ (positive and negative mitotic signals). Both cytokines can promote and inhibit chemotaxis and cell growth [27], and their behavior depends on the context.

In this study, the correlations between the PDGF-AA level and the clinical and laboratory parameters in DM patients had the same pattern as FGF-1 with the exception of the cutaneous manifestations. FGF-1 has been shown to induce the expression of PDGF-AA in endothelial cells through an unknown mechanism [28]. Thus, 
in active DM, these cytokines may interact in a synergistic manner to potentiate their angiogenic actions through interactions with their receptors.

One possible explanation for the reduction in the VEGF serum levels in DM patients is that most of our patients were in an early stage of the disease, were well treated and were controlled symptomatically; therefore, little inflammatory infiltration was present and the capillary efficiency was more established. Another probable hypothesis is that the release of this cytokine by endothelial and inflammatory cells occurred initially at sites of greater inflammation, such as the muscle and/or skin, without the need for blood transport [10]. Thus, local measurement of VEGF in these tissues and an analysis of its gene expression patterns are needed in future studies.

The serum PDGF- BB, ANG and ANGPT-1 levels were correlated with the DM disease parameters, similar to FGF-1.

As discussed above, the PDGFs interact with specific receptors, and PDGF-BB has an affinity for all PDGF-R heterodimers [27]. Similarly, other cytokines, such as FGF-2, have an activation/inhibition relationship with PDGF [29]. The cytokine profile found in this study shows an increase of FGF-2 in DM patients, which may have inhibitory effects on PDGF-BB.

In agreement with our results, only one study evaluated the serum ANG levels in DM patients [8] and found no significant difference in the ANG levels between groups. However, the authors observed high gene expression of ANG in the analysed skin biopsies which suggests in situ inflammatory activity control more than a systemic control.

ANGPT-1 also interacts with other cytokines, such as VEGF, on endothelial cells to inhibit leukocyte adhesion the expression of some specific cell adhesion molecules [30]. Another important antagonist of ANGPT-1 is ANGPT-2 [31]. The interaction with inhibitory factors, such as ANGPT-2, may allow the serum levels to remain similar the levels observed in healthy people, as shown in our results.

Considering that the cytokines with the highest serum levels evaluated in this work were FGF-1 and FGF-2 and the correlation of these cytokine levels with the treatment received, we cannot exclude the possibility that the immunosuppressive treatment may have influenced the results by interfering with the inflammatory and angiogenic processes.

Among the limitations of the present study were the transversal nature and the analysis of the serum levels of the cytokines performed in a single measurement, because the serum profiles could have changed during the evolution of the disease and the establishment of treatment.

\section{Conclusions}

In summary, we found an increase in the serum FGF-1 and FGF-2 levels, a decrease in the VEGF and PDGF-AA levels, and comparable ANG, ANGPT-1 and PDGF-BB levels in patients with $\mathrm{DM}$ and the healthy controls. The FGF-1, PDGF-AA, ANG and AGPT-1 levels showed a positive correlation with the disease activity parameters, which was in contrast to the FGF-2 level. Moreover, FGF-1 and FGF-2 correlated positively with the cutaneous DM manifestations and cumulative prednisone dose, respectively. These data provide the possible involvement of angiogenic cytokines in DM disease.

\section{Abbreviations}

ALT: Alanine aminotransferase; ANG: Angiogenin; ANGPT: Angiopoietin; AST: Aspartate aminotransferase; BMI: Body mass index; CPK: Creatine phosphokinase; DM: Dermatomyositis; FGF: Fibroblast growth factor; HAQ: Health Assessment Quality; LDH: Lactate dehydrogenase; MMT: Manual muscle testing; PDGF: Platelet derived growth factor; SD: Standard deviation; VAS: Visual analogue scale; VEGF: Vascular endothelial growth factor

\section{Support}

Federico Foundation; Fundação Faculdade de Medicina; Fundação de Amparo à Pesquisa do Estado de São Paulo (FAPESP) (\#2012/07101-4 and \#2012/09633-3) to SKS; Coordenação de Aperfeiçoamento de Pessoal de Nível Superior (CAPES) to MGS.

\section{Authors' contributions \\ All authors contributed equally to write and review the manuscript. All authors read and approved the final manuscript.}

\section{Ethics approval and consent to participate}

The study was approved by the local ethics committee (HCFMUSP CAPPesq number 1.545.393) and all patients signed the informed consent form.

\section{Consent for publication}

Not applicable.

\section{Competing interests}

All authors declare that they have no conflicts of interest.

\section{Publisher's Note}

Springer Nature remains neutral with regard to jurisdictional claims in published maps and institutional affiliations.

Received: 26 April 2018 Accepted: 2 July 2018

Published online: 31 July 2018

\section{References}

1. Bohan A, Peter JB. Polymyositis and dermatomyositis (first of two parts). N Engl J Med. 1975;292:344-7.

2. Bohan A, Peter JB. Polymyositis and dermatomyositis (second of two parts). N Engl J Med. 1975;292:403-7.

3. Dimachkie MM, Barohn RJ, Amato A. Idiopathic inflammatory myopathies. Neurol Clin. 2014;32:595-628.

4. Dalakas MC. Inflammatory muscle diseases. N Engl J Med. 2015;373:393-4.

5. Dalakas MC. Pathophysiology of inflammatory and autoimmune myopathies. Presse Med. 2011:40:e237-47.

6. Dalakas MC. Review: an update on inflammatory and autoimmune myopathies. Neuropathol Appl Neurobiol. 2011;37:226-42.

7. Orlandi M, Barsotti S, Cioffi E, Tenti S, Toscano C, Baldini C, et al. One year in review 2016: idiopathic inflammatory myopathies. Clin Exp Rheumatol. 2016; 34:966-74.

8. Kuwahara A. Angiogenin expression in the sera and skin of patients with rheumatic diseases. Biosci Trends. 2012;6:229-33. 
9. Kikuchi K, Kubo M, Kadono T, Yazawa N, Ihn H, Tamaki K. Serum concentrations of vascular endothelial growth factor in collagen diseases. $\mathrm{Br}$ J Dermatol. 1998;139:1049-51.

10. Grundtman C, Tham E, Ulfgren AK, Lundberg IE. Vascular endothelial growth factor is highly expressed in muscle tissue of patients with polymyositis and patients with dermatomyositis. Arthritis Rheum. 2008:58:3224-38.

11. Volpi N, Pecorelli A, Lorenzoni P, Di Lazzaro F, Belmonte G, Agliano M, et al. Antiangiogenic VEGF isoform in inflammatory myopathies. Mediat Inflamm. 2013;2013:219313

12. Ishikawa A, Okada J, Nishi K, Hirohata S. Efficacy of serum angiopoietin-1 measurement in the diagnosis of early rheumatoid arthritis. Clin Exp Rheumatol. 2011;29:604-8.

13. Kadono T, Kikuchi K, Kubo M, Fujimoto M, Tamaki K. Serum concentrations of basic fibroblast growth factor in collagen diseases. J Am Acad Dermatol. 1996;35:392-7.

14. Carvalho JF, Blank M, Shoenfeld Y. Vascular endothelial growth factor (VEGF) in autoimmune diseases. J Clin Immunol. 2007;27:246-56.

15. Rider LG, Giannini EH, Harris-Love M, Joe G, Isenberg D, Pilkington C, et al. Defining clinical improvement in adult and juvenile myositis. J Rheumatol. 2003;30:603-17.

16. Miller FW, Rider GL, Chung YL, Cooper R, Danko K, Farewell V, et al. Proposed preliminary core set measures for disease outcome assessment in adult and juvenile idiopathic inflammatory myopathies. Rheumatology (Oxford). 2001;40:1262-73.

17. Isenberg DA, Allen E, Farewell V, Ehrenstein MR, Hanna MG, Lundberg IE, et al. International myositis and clinical studies group (IMACS). International consensus outcome measures for patients with idiopathic inflammatory myopathies. Development and initial validation of myositis activity and damage indices in patients with adult onset disease. Rheumatology (Oxford). 2004;43:49-54.

18. Rider LG, Koziol D, Giannini EH, Jain MS, Smith MR, Whitney-Mahoney K, et al. Validation of manual muscle testing and a subset of eight muscles for adult and juvenile idiopathic inflammatory myopathies. Arthritis Care Res. 2010;62:465-72.

19. Bruce B, FrieS JF. The Stanford health assessment questionnaire: dimensions and practical applications. Health Qual Life Outcomes. 2003;1:20.

20. Sada KE, Yamasaki Y, Maruyama M, Sugiyama H, Yamamura M, Maeshima $Y$, et al. Altered levels of adipocytokines in association with insulin resistance in patients with systemic lupus erythematosus. J Rheumatol. 2006;33:1545-52.

21. Dignass AU, Tsunekawa S, Podolsky DK. Fibroblast growth factors modulate intestinal epithelial cell growth and migration. Gastroenterol. 1994;106: 1254-62.

22. Holland EC, Varmus HE. Basic fibroblast growth factor induces cell migration and proliferation after glia- specific gene transfer in mice. Proc Nat Acad Sci USA. 1998;95:1218-23.

23. Hossain WA, Morets DK. Fibroblast growth factors (FGF-1. FGF-2) promote migration and neurite growth of mouse cochlear ganglion cells in vitro: immunohistochemistry and antibody perturbation. J Neurosci Res. 2000;62: 40-55.

24. Yun YR, Won JE, Jeon E, Lee S, Kang W, Jo H, et al. Fibroblast growth factors: biology, function and application for tissue regeneration. J Tissue Eng. 2010;218142:2010

25. Broadley KN, Aquino AM, Hicks B, Ditesheim JA, McGee GS, Demetriou AA, et al. The diabetic rat as an impaired wound healing model: stimulatory effects of transforming growth factor-beta and basic fibroblast growth factor. Biotechnol Ther. 1989;1:55-68.

26. Yablonka-Reuveni Z, Seger R, Rivera AJ. Fibroblast growth factor promotes recruitment of skeletal muscle satellite cells in young and old rats. J Histoch Cytoch. 1999;47:23-42.

27. Tengood JE, Ridenour R. BrodskYR, Russell AJ, little SR. sequential delivery of basic fibroblast growth factor and platelet-derived growth factor for angiogenesis. Tissue Eng. Part. 2011;7:1181-9.

28. Delbridge GJ, Khachigian LM. FGF-1 induced platelet-derived growth factor-a chain gene expression in endothelial cells involves transcriptional activation by early growth response factor-1. Circ Res. 1997;81:282-8.

29. Facchiano A, De Marchis F, Turchetti E, Facchiano F, Guglielmi M, Denaro A, et al. The chemotactic and mitogenic effects of platelet-derived growth factor-BB on rat aorta smooth muscle cells are inhibited by basic fibroblast growth factor. J Cell Sci. 2000;113:2855-63.
30. Fukuhara S, Sako K, Noda K, Zhang J, Minami M, Mochizuki N. Angiopoietin1/Tie2 receptor signaling in vascular quiescence and angiogenesis. Histol Histopathol. 2010;25:387-96.

31. Kim I, Moon SO, Park SK, Chae SW, Koh GY. Angiopoietin-1 reduces VEGFstimulated leukocyte adhesion to endothelial cells by reducing ICAM-1. VCAM-1 and E-selectin expression. Circ Res. 2001;89:477-9.

\section{Ready to submit your research? Choose BMC and benefit from:}

- fast, convenient online submission

- thorough peer review by experienced researchers in your field

- rapid publication on acceptance

- support for research data, including large and complex data types

- gold Open Access which fosters wider collaboration and increased citations

- maximum visibility for your research: over $100 \mathrm{M}$ website views per year

At BMC, research is always in progress.

Learn more biomedcentral.com/submissions 